\title{
BOUNDARY VALUES OF HOLOMORPHIC FUNCTIONS
}

BY ELIAS M. STEIN

Communicated by J. J. Kohn, May 18, 1970

Let $D$ be a bounded domain in $C^{n}$ with smooth boundary. We shall consider the behavior near the boundary of holomorphic functions in D. Our results are of two kinds: those valid without any further restriction on $D$, and those which require that $D$ is strictly pseudoconvex. Detailed proofs will appear in [7].

1. Fatou's theorem and $H^{p}$ spaces. We assume that $D$ is a bounded domain with smooth boundary. We first define the appropriate approach to the boundary which extends the usual nontangential approach and takes into account the complex structure of $\mathbf{C}^{n}$. Let $w \in \partial D$, and let $\nu_{w}$ be the unit outward normal at $w$. For each $\alpha>0$ consider the approach region $a_{\alpha}(w)$ defined by

$\mathfrak{a}_{\alpha}(w)=\left\{z \in \mathbb{D}:\left|\left(z-w, \nu_{w}\right)\right|<(1+\alpha) \delta_{w}(z),|z-w|^{2}<\alpha \delta_{w}(z)\right\}$.

Here $(z, w)=z_{1} \bar{w}_{1}+\cdots+z_{n} \bar{w}_{n},|z|^{2}=(z, z)$, and $\delta_{w}(z)$ denotes the minimum of the distances of $z$ from $\partial D$ and from $z$ to the tangent hyperplane to $\partial D$ at $w$.

We shall say that $F$ is admissibly bounded at $w$ if $\sup _{\boldsymbol{z} \in \mathrm{a}_{\alpha}(w)}|F(z)|$ $<\infty$, for some $\alpha ; F$ has an admissible limit at $w$, if $\lim _{z \rightarrow w}, x \in a_{\alpha}(w) F(z)$ exists, for all $\alpha>0$. On $\partial D$ we shall take the measure induced by Lebesgue measure on $\boldsymbol{C}^{n}$; we denote it by $m(\cdot)$, or $d \sigma$. The extension of the classical Fatou theorem is as follows.

TheOREM 1. Suppose $F$ is holomorphic and bounded in D. Then $F$ has an admissible limit at almost every $w \in \partial D$.

Note. This is stronger than the usual nontangential approach one would obtain using the theory of harmonic functions in $R^{2 n}$. As is to be observed, the admissible approach allows a parabolic tangential approach in directions corresponding to $2 n-2$ real dimensions.

We consider two types of balls on $\partial \mathscr{D}$. For any $\rho>0$ and $w \in \partial D$,

(1) $B_{1}(w, \rho)=\left\{w^{\prime} \in \partial D:\left|w-w^{\prime}\right|<\rho\right\}$;

(2) $B_{2}(w, \rho)=\left\{w^{\prime} \in \partial D:\left|\left(w-w^{\prime}, \nu_{w}\right)\right|<\rho,\left|w-w^{\prime}\right|^{2}<\rho\right\}$. Observe that $m\left(B_{1}(w, \rho)\right) \sim c_{1} \rho^{2 n-1}$, and $m\left(B_{2}(w, \rho)\right) \sim c_{2} \rho^{n}$ as $\rho \rightarrow 0$.

AMS 1969 subject classifications. Primary 3217, 3111; Secondary 3220.

Key words and phrases. Fatou's theorem, strictly pseudo-convex domains, maximal functions, $H^{p}$ spaces, Kähler metric. 
TheOREM 3. Suppose $F \in N$. Then $F$ has admissible limits at almost every $w \in \partial D$.

We consider the following related maximal functions defined for function on $\partial D$ :

$$
f_{j}^{*}(w)=\sup _{\rho>0} \frac{1}{m\left(B_{j}(w, \rho)\right)} \int_{B_{j}(w, \rho)}\left|f\left(w^{\prime}\right)\right| d \sigma\left(w^{\prime}\right), \quad j=1,2 .
$$

Then the $f_{j}^{*}$ satisfy the usual inequalities for maximal functions. (For $j=1$, see e.g. K. T. Smith [4]; for $j=2$, see e.g. Hörmander [1], or Stein [6], and the works cited there.) We define $M f$ to be the superposition of these two, i.e. $M(f)(w)=\left(f_{1}^{*}\right)_{2}^{*}(w)$. The main step in the proof of Theorem 1 is an argument of harmonic majorization which is essentially contained in the following lemma.

Lemma. Suppose $u$ is continuous in $\bar{D}$ and pluri-subharmonic in $D$. Let $f$ be the restriction of $u$ to $\partial D$. Then for each $\alpha>0$

$$
\sup _{z \in a_{\alpha}(w)}|u(z)| \leqq C_{\alpha} M f(w) .
$$

The same argument also allows an extension to $H^{p}$ spaces. Suppose $\lambda(z)$ is a smooth real-valued function on $C^{n}$, so that $D=\{z: \lambda(z)<0\}$, and $\left|\nabla \lambda\left(z^{0}\right)\right|>0$, whenever $\lambda\left(z^{0}\right)=0$. For sufficiently small $\epsilon$ consider the approximating regions $D_{\mathrm{e}}$ defined by $D_{\mathrm{e}}=\{z: \lambda(z)<-\epsilon\}$. If $0<p<\infty$, and $F$ is holomorphic in $D$, we say that $F \in H^{p}(D)$ if

$$
\sup _{\bullet>0} \int_{\partial D_{\epsilon}}|F(z)| p d \sigma_{\epsilon}(z)<\infty .
$$

$d \sigma_{\epsilon}$ is the measure on $\partial D_{\epsilon}$ induced by Lebesgue measure in $C^{n}$. It can be shown that the property that $F \in H^{p}(D)$ is independent of the particular approximating regions $D_{\mathbb{E}}$ defined above, and is thus intrinsic. It is equivalent with the fact that $|F|^{p}$ has a harmonic majorant in D. ("Harmonic" is taken in the usual sense in $R^{2 n}$.)

Theorem 2. Suppose $F \in H^{p}(D)$. Then

(a) $\quad \int_{\partial D} \sup _{z \in Q \alpha(w)}|F(z)|^{p} d \sigma(w) \leqq A_{p, \alpha} \sup _{\iota>0} \int_{\partial D_{e}}|F(z)|^{p} d \sigma_{e}(z)$;

(b) F has an admissible limit at almost every $w \in \partial D$.

There is an analogue also for the Nevanlinna class $N$. This class is defined as all holomorphic functions $F$ in $D$ for which

$$
\sup _{\boldsymbol{e}>0} \int_{\partial D_{e}} \log ^{+}|F(z)| d \sigma_{\epsilon}(z)<\infty .
$$


The proof of Theorem 3 requires a modification of estimate (3) of Lemma 1 , where $M$ is replaced by a variant which is finite almost everywhere whenever $f \in L^{1}(d \sigma){ }^{1}$

2. Local Fatou theorem and area integral. From now on we shall assume that in addition $D$ is strictly pseudo-convex. We shall introduce a potential theory in $D$ which reflects this property in an intimate way. This will be done in terms of a Kähler metric which we now construct in terms of the geometry of $\partial D$. For every $z \in D$ sufficiently close to $\partial D$, let $n(z)$ denote the normal projection of $z$ on $\partial \mathcal{D}$. Then the mapping $z \rightarrow n(z)$ is smooth. For $z$ near $\partial D$ we let $\nu_{z}$ denote the (outward) unit normal at $n(z)$. This induces a direct sum decomposition $\boldsymbol{C}^{n}=N_{z} \oplus C_{z}$, where $N_{z}=\left\{C \nu_{z}\right\}$ and $C_{z}=\left(N_{z}\right)^{\perp}$; the orthogonal complement is taken with respect to the usual (complex) inner product $(\cdot, \cdot)$ on $C^{n} . N_{z}$ and $C_{z}$ have complex dimension 1 and $n-1$ respectively.

Lemma 2. There exists a Kähler metric $d s^{2}=\sum g_{i j}(z) d z_{i} d \bar{z}_{j}$ defined on $D$ with the following properties:

(a) The $g_{i j}(z)$ are smooth on D.

(b) $\sum_{i, j} g_{i j}(z) \zeta_{i} \bar{\zeta}_{j} \approx(\delta(z))^{-2}|\zeta|^{2}$, for $\zeta \in N_{z}$.

(c) $\sum_{i, j} g_{i j}(z) \zeta_{i} \bar{\zeta}_{j} \approx(\delta(z))^{-1}|\zeta|^{2}$, for $\zeta \in C_{z}$.

(d) $\left|\sum_{i, j} g_{i j}(z) \zeta_{i} \bar{\zeta}_{j}^{\prime}\right| \leqq c(\delta(z))^{-1}|\zeta|\left|\zeta^{\prime}\right|$, for $\zeta \in C_{z}$, and $\zeta^{\prime} \in N_{z}$. $\delta(z)$ denotes the distance of $z$ from $\partial D$ D.

One choice of the metric $g_{i j}$ is the one given near the boundary by

$$
g_{i j}(z)=\frac{\partial^{2}}{\partial z_{i} \partial \bar{z}_{j}}[\log 1 / \delta(z)] .
$$

With this metric we form the Laplace-Beltrami operator $\Delta$ which is given by

$$
\Delta=4 \sum_{i, j} g^{i j} \frac{\partial^{2}}{\partial \bar{z}_{i} \partial z_{j}}
$$

where $\left\{g^{i j}\right\}$ is the inverse matrix to $\left\{g_{i j}\right\}$. This Laplace operator is elliptic in $D$ but degenerates at the boundary in a way which takes into account the strict pseudo-convexity of $\partial D$. We study the potential theory for the Kähler manifold $D$ with the above metric and Laplace operator $\Delta$, by applying Green's theorem in this set up. The following lemma is needed to carry this out.

1 The argument at this stage was suggested to me by C. L. Fefferman. 
LEMMA 3. For $z$ near the boundary $\left|\Delta\left[(\delta(z))^{n}\right]\right| \leqq c(\delta(z))^{n+1}$.

The thrust of the lemma is that $(\delta(z))^{n}$ is approximately "harmonic" with respect to $\Delta$. In effect $(\delta(z))^{n}$ plays the role near the boundary that $\log 1 /|z|$ plays near $|z|=1$ in the case $n=1$, when $D$ is the unit disc.

To state the main result we define the analogue of the area integral. Let $|\nabla F|^{2}$ denote the square of the norm of the gradient (taken with respect to the metric $d s^{2}$ above) for holomorphic $F$. Thus

$$
|\nabla F|^{2}=2 \sum_{i, j} g^{i j} \frac{\overline{\partial f}}{\partial z_{i}} \frac{\partial f}{\partial z_{j}} .
$$

For any $\alpha>0$ we define

$$
S(F)(w)=\left(\int_{a_{\alpha}(w)}|\nabla F(z)|^{2} d \Omega(z)\right)^{1 / 2}
$$

where $d \Omega$ is the element of volume induced by the metric $d s^{2}$. In order to see the meaning of the above suppose for simplicity that $w=0$, and the unit normal $\nu_{w}$ is along the positive $y_{1}$ direction, $z_{1}=x_{1}+i y_{1}$. Then in $a_{\alpha}(w)$

$$
|\nabla F|^{2} \approx y_{1}^{2}\left|\frac{\partial F}{\partial z_{1}}\right|^{2}+y_{1} \sum_{k=2}^{n}\left|\frac{\partial F}{\partial z_{k}}\right|^{2},
$$

and $d \Omega \approx y_{1}^{-n-1} d z$, where $d z$ denotes Lebesgue measure in $C^{n}$.

ThEOREM 4. Suppose $F$ is holomorphic in D. Then at almost every $w \in \partial D$ the following properties are equivalent:

(a) $F$ is admissibly bounded at w.

(b) F has an admissible limit at w.

(c) $S(F)(w)<\infty$.

The idea of the proof is to show that almost everywhere $(a) \Rightarrow(c)$, and $(c) \Rightarrow(b)$. To prove $(a) \Rightarrow(b)$ we use the analogue of the argument involving Green's theorem we gave in [5], but now for the potential theory constructed above. To prove $(c) \Rightarrow(b)$ we show first that the finiteness of $S(F)$ implies the finiteness of the standard "area integral", thus implying nontangential convergence for almost every point in question. Secondly, condition (c) can also be used as a Tauberian condition, refining nontangential to admissible convergence. 


\section{REFERENCES}

In addition to the works already quoted the papers below have a direct bearing on this research.

1. L. Hörmander, $L^{p}$ estimates for (phuri-) subharmonic functions, Math. Scand. 20 (1967), 65-78. MR 38 \#2323.

2. A. Koranyi, Harmonic functions on Hermitian hyperbolic space, Trans. Amer. Math. Soc. 135 (1969), 507-516.

3. A. Koranyi and E. M. Stein, Fatou's theorem for generalized half-planes, Ann. Scuola Norm. Sup. Pisa 22 (1968), 107-112.

4. K. T. Smith, $A$ generalization of an inequality of Hardy and Littlewood, Canad. J. Math. 8 (1956), 157-170. MR 19, 261.

5. E. M. Stein, On the theory of harmonic functions of several variables. II: Behavior near the boundary, Acta Math. 106 (1961), 137-174. MR 30 \#3234.

6. - The analogues of Fatou's theorem and estimates for maximal functions, Geometry of Homogeneous Bounded Domains (C.I.M.E., $3^{\circ}$ Ciclo, Urbino, 1967) Edizioni Cremonese, Rome, 1968, pp. 291-307. MR 38 \#3466.

7. - Boundary of holomorphic functions of several variables, Lecture Notes by W. Beckner, Princeton University, 1970. (to appear).

8. K-O. Widman, On the boundary behavior of solutions to a class of elliptic partial differential equations, Ark. Mat. 6 (1966), 485-533. MR 36 \#2949.

Princeton University, Princeton, New Jersey 08540 